\title{
Tryptophan fluorescence quenching in $\beta$-lactam-interacting proteins is modulated by the structure of intermediates and final products of the acylation reaction
}

Sébastien Triboulet ${ }^{\mathrm{a} 1}$, Zainab Edoo ${ }^{\mathrm{a} 1}$, Fabrice Compain ${ }^{\mathrm{a}, \mathrm{b}}$, Clément Ourghanliana, Adrian Dupuis ${ }^{\mathrm{a}}$, Vincent Dubée ${ }^{a}$, Laetitia Sutterlina, Heiner Atzea, Mélanie Ethève-Quelquejeuc, Jean-Emmanuel Hugonnet $^{\mathrm{a} 2}$, and Michel Arthur ${ }^{\mathrm{a} 2}$

${ }^{1}$ Equal contribution

${ }^{2}$ Equal contribution and corresponding authors

[a] Dr. S Triboulet, Ms Z Edoo, Dr. F Compain, Dr. C Ourghanlian, Mr A Dupuis, Dr. V Dubée, Dr. L Sutterlin, Dr. H Atze, Dr. JE Hugonnet, Dr. M Arthur, Sorbonne Université, Sorbonne Paris Cité, Université Paris Descartes, Université Paris Diderot, INSERM, Centre de Recherche des Cordeliers, CRC, F-75006 Paris, France. E-mail: jean-emmanuel.hugonnet@crc.jussieu.fr; michel.arthur@crc.jussieu.fr

[b] Dr. F Compain, Service de Microbiologie, Assistance Publique-Hôpitaux de Paris, Hôpital Européen Georges Pompidou, Paris, France.

[c] Dr. M Ethève-Quelquejeu, Laboratoire de Chimie et de Biochimie Pharmacologiques et Toxicologiques, Université Paris Descartes, UMR 8601, Paris, F-75005 France; CNRS UMR 8601, Paris, F-75006 France.

Keywords: acylenzyme, $\beta$-lactamase, fluorescence quenching, L,D-transpeptidase, tryptophan fluorescence. 
In most bacteria, $\beta$-lactam antibiotics inhibit the last cross-linking step of peptidoglycan synthesis by acylation of the active-site Ser of D,D-transpeptidases belonging to the penicillin-binding protein (PBP) family. In mycobacteria, cross-linking is mainly ensured by L,D-transpeptidases (LDTs), which are promising targets for the development of $\beta$-lactam-based therapies for multidrug-resistant tuberculosis. For this purpose, fluorescence spectroscopy is used to investigate the efficacy of LDT inactivation by $\beta$-lactams but the basis for fluorescence quenching during enzyme acylation remains unknown. In contrast to what has been reported for PBPs, we show here using a model L,Dtranspeptidase $\left(\mathrm{Ldt}_{\mathrm{fm}}\right)$ that fluorescence quenching of Trp residues does not depend upon direct hydrophobic interaction between Trp residues and $\beta$-lactams. Rather, Trp fluorescence was quenched by the drug covalently bound to the active-site Cys residue of $\mathbf{L d t}_{\mathrm{fm}}$. Fluorescence quenching was not quantitatively determined by the size of the drug and was not specific of the thioester link connecting the $\beta$-lactam carbonyl to the catalytic $C y s$ as quenching was also observed for acylation of the active-site Ser of $\beta$-lactamase BlaC from $M$. tuberculosis. Fluorescence quenching was extensive for reaction intermediates containing an amine anion and for acylenzymes containing an imine stabilized by mesomeric effect, but not for acylenzymes containing a protonated $\beta$-lactam nitrogen. Together, these results indicate that the extent of fluorescence quenching is determined by the status of the $\beta$-lactam nitrogen. Thus, fluorescence kinetics can provide information not only on the efficacy of enzyme inactivation but also on the structure of the covalent adducts responsible for enzyme inactivation.

Peptidoglycan is an essential constituent of bacterial cell walls since it prevents cell swelling and lysis by mechanically sustaining the osmotic pressure of the cytoplasm ${ }^{1}$. This protective function depends upon synthesis and maintenance during the entire cell cycle of the net-like peptidoglycan macromolecule, which completely surrounds the bacterial cell. Peptidoglycan is made of glycan strands cross-linked by short peptide stems. In most bacteria, the cross-linking step is performed by D,Dtranspeptidases, which are the essential targets of $\beta$-lactam antibiotics and are often referred to as penicillin-binding proteins (PBPs) ${ }^{2}$. In mycobacteria ${ }^{3-4}$ and in Clostridium difficile ${ }^{5-6}$, the cross-links found are mainly (70\% to $80 \%$ ) formed by a second class of enzymes, the L,D-transpeptidases (LDTs). Since LDTs are not inhibited by $\beta$-lactams belonging to the penam class, such as ampicillin, ${ }^{7-8}$, these enzymes are responsible for high-level resistance to these drugs in mutants of Enterococcus faecium ${ }^{9-}$ ${ }^{10}$ and Escherichia coli ${ }^{11}$ selected in laboratory conditions.

PBPs and LDTs are structurally unrelated ${ }^{12-15}$ and proceed through different catalytic mechanisms for activation of Ser and Cys nucleophiles ${ }^{16}$, which are part of Lys-Ser ${ }^{17}$ and Cys-His-Asp ${ }^{18}$ catalytic diad and triad, respectively. PBPs and LDTs also differ by the structure of the stem peptide used as an acyl donor, a pentapeptide for PBPs ${ }^{17}$ and a tetrapeptide for LDTs ${ }^{16}$, except for Enterococcus faecalis $\mathrm{Ldt}_{\mathrm{fs}}{ }^{19}$. PBPs cleave the D-Ala ${ }^{4}-\mathrm{D}-\mathrm{Ala}{ }^{5}$ peptide bond of the acyl donor, hence the $\mathrm{D}, \mathrm{D}$ designation for these transpeptidases, and link the carbonyl of $\mathrm{D}-\mathrm{Ala}{ }^{4}$ to the side-chain amino group of the $3^{\text {rd }}$ residue thereby generating $4 \rightarrow 3$ cross-links ${ }^{17}$ (Supplementary Fig. S1). In contrast, LDTs cleave the L-Lys ${ }^{3}$-D-Ala ${ }^{4}$ bond of the donor (L,D designation) and form $3 \rightarrow 3$ cross-links ${ }^{16}$.

The first substrate of the transpeptidation reaction, the acyl donor, forms an acylenzyme intermediate that subsequently reacts with the second substrate, the acceptor, leading to the final cross-linked product. For PBPs, nucleophilic attack of the carbonyl of D-Ala ${ }^{4}$ by the active-site Ser results in an acylenzyme containing an ester bond and release of D-Ala ${ }^{517}$. For the acylenzyme formed by LDTs, a thioester bond connects the carbonyl of L-Lys ${ }^{3}$ to the $\gamma$ sulfur of the active-site Cys ${ }^{16}$. $\beta$ - 
lactams are structure analogues of the D-Ala ${ }^{4}$-D-Ala ${ }^{5}$ extremity of stem pentapeptides and act as suicide substrates of the PBPs ${ }^{20}$. Nucleophilic attack of the carbonyl of the $\beta$-lactam ring by the active-site Ser of PBPs leads to formation of an acylenzyme, which is only hydrolyzed very slowly, leading in practice to "irreversible" inactivation in the time scale of a bacterial generation (Supplementary Fig. S1) ${ }^{2}$. LDTs are also acylated by $\beta$-lactams although efficacious enzyme inactivation and antibacterial activity occur only for a single class of $\beta$-lactams, the carbapenems, such as imipenem ${ }^{7-8} . \beta$-lactams of the cephem (cephalosporin) class, such as ceftriaxone, are only active at high concentrations since acylation is slower and the resulting thioester bond is prone to hydrolysis ${ }^{7}$. The kinetic parameters are even less favorable for penams (ampicillin and penicillin) leading to equilibrium between the acylated and unacylated (functional) forms, which accounts for the lack of antibacterial activity ${ }^{7}$.

Fluorescence kinetics revealed that the acylation reaction of LDTs by $\beta$-lactams of the carbapenem class comprises two limiting steps (Fig. 1A and B). In the first step, nucleophilic attack of the $\beta$-lactam carbonyl by the negatively charged sulfur atom of the catalytic Cys was thought to lead to formation of a covalent sulfur-carbon bond and to the development of a negative charge on the drug ${ }^{21}$. Initially, this negative charge was proposed to be located on the $\beta$-lactam oxygen ${ }^{21}$. This hypothesis was based on analogies with PBPs, which contain an oxyanion hole for stabilization of the negative charge developing on the $\beta$-lactam oxygen ${ }^{17}$. However, hybrid potential simulation of the acylation reaction indicated that the path to an oxyanion is energetically disfavored in the case of LDTs and that an oxyanion could not correspond to a stabilized reaction intermediate ${ }^{22}$. Rather, hybrid potential simulation indicated that the most energetically favored reaction path involves a concerted mechanism leading to the concomitant formation of the thioester bond, rupture of $\mathrm{C}-\mathrm{N} \beta$-lactam bond, and formation of an amine anion ${ }^{22}$. In the second step, the final acylenzyme is formed by protonation of the amine anion. The origin of this proton remains elusive ${ }^{18,22}$. Formation of a non-covalent complex is not considered in this reaction scheme as the affinity of the $\mathrm{Ldt}_{\mathrm{fm}}$ for carbapenems is very low, in the order of 60 to $80 \mathrm{mM}^{7}$.

Fluorescence kinetics proved useful to assess the efficacy of inactivation of LDTs from Mycobacterium tuberculosis ${ }^{23-25}$ and contributed to the choice of the drugs that were tested in a phase II clinical trial ${ }^{26}$. The assays were also useful in evaluating series of synthetic carbapenems ${ }^{27}$ and non$\beta$-lactam LDT inhibitors ${ }^{28}$. However, the basis for the variation in fluorescence intensity detected during the two steps of the acylation reaction remained elusive. Initially, we developed fluorescence kinetics since determination of the crystal structure of $L_{d t} t_{f m}$ revealed a Trp residue at the entrance of the catalytic cavity ${ }^{15}$, which appeared ideally located for fluorescence quenching upon drug binding due to changes in its environment ${ }^{21}$. However, this naïve explanation was subsequently found to be insufficient to account for the various behaviors observed for acylation of LDTs by representatives of the $\beta$-lactam classes ${ }^{7,22-25}$. This prompted us to investigate here the basis for variations in fluorescence intensity occurring upon acylation of LDTs using the well-characterized L,D-transpeptidase Ldt $_{\mathrm{fm}}$ from E. faecium as a model. We show that variations in fluorescence intensity are not due to modification of the environment of Trp residues but to the formation of a $\beta$-lactam-derived fluorescence quencher during the acylation reaction.

\section{RESULTS}

Trp to Phe and His to Ala substitutions do not abolish the biphasic behavior of fluorescence kinetics observed for $\mathbf{L d t}_{\mathrm{fm}}$ inactivation by imipenem. We have previously proposed that the acylation of $\mathrm{Ldt}_{\mathrm{fm}}$ by carbapenems is a two-step reaction involving reversible formation of an amine anion (EIA, step 1) 
followed by irreversible formation of an acylenzyme (EI*, step 2) (Fig. 1A) ${ }^{21-22}$. Fluorescence kinetics also display two phases enabling the determination of kinetic parameters for the two steps of the reaction (Fig. 1B) ${ }^{21}$. In the first phase, fluorescence intensity decreases because $\mathrm{EI}^{\mathrm{An}}$ is rapidly formed to the detriment of the free enzyme $(\mathrm{E})$ and the fluorescence intensity of $\mathrm{El}^{\mathrm{An}}$ is lower than that of $\mathrm{E}$. In the second phase, fluorescence intensity increases since the acylenzyme $\left(\left.\mathrm{E}\right|^{*}\right)$ is formed to the detriment of $\mathrm{El}^{\mathrm{An}}$ (acylation step) and the fluorescence intensity of $\mathrm{El}^{*}$ is greater than that of $\mathrm{El}^{\mathrm{An}}$. Our first aim was to determine whether variations in the fluorescence intensity could be correlated to the modification of the environment of a specific Trp residue of $L_{d t_{f m}}$. To address this question, Trp residues of the catalytic domain of $\mathbf{L d t}_{\mathrm{fm}}$ were replaced by Phe residues in various combinations. For the sake of simplicity, the six Trp residues at positions $355,385,410,415,425$, and 434 of $^{2} \mathrm{Ldt}_{\mathrm{fm}}$ were designated as residues a to $\mathrm{f}$, respectively. Fluorescence kinetics were determined for $\mathrm{Ldt}_{\mathrm{fm}}$ derivatives lacking 2 to 5 Trp residues (Fig. 1C). Fluorescence kinetics remained bi-phasic in all cases, except for the $\mathrm{Ldt}_{\mathrm{fm}}$ derivative only retaining the "a" residue (i.e. $\operatorname{Tr} \mathrm{T}^{355}$ ). Thus, the variations in the fluorescence intensity associated with the two steps of the acylation reaction do not depend upon the modification of the environment of a specific Trp residue of $\mathrm{Ldt}_{\mathrm{fm}}$. This prompted us to investigate fluorescence quenching by His residues of $\mathrm{Ldt}_{\mathrm{fm}}$. Fluorescence kinetics obtained with the $\mathrm{His}^{353} \mathrm{Ala}$ and $\mathrm{His}^{440} \mathrm{Ala}$ derivatives of $\mathrm{Ldt}_{\mathrm{fm}}$ indicated that neither residue is essential for biphasic fluorescence kinetics (Fig. 1C). His at position 421 could not be analyzed since this residue is part of the catalytic triad and is essential for $L \mathrm{Lt}_{\mathrm{fm}}$ activity.

Impact of Trp to Phe and His to Ala substitutions on the efficacy of the inactivation reaction and on the relative fluorescence intensity of the three enzyme forms. Fitting simulations to experimental data for four concentrations of imipenem was used to determine the inactivation kinetic parameters (Supplementary Fig. S2). None of the Trp residues was essential for the acylation of $\mathrm{Ldt}_{\mathrm{fm}}$ by imipenem although important variations were observed in the $k_{1}$ (formation of the amine anion) and $k_{2}$ (acylation step) parameters (Fig. 2A and 2B, respectively). The Trp to Phe substitutions produced large variations in the intrinsic fluorescence of the three forms of the enzyme (apo $\mathrm{Ldt}_{\mathrm{fm}}$, amine anion, and acylenzyme; Fig. $2 \mathrm{C}$ ). The formation of the acylenzyme (El*) was confirmed by mass spectrometry for all $\mathrm{Ldt}_{\mathrm{fm}}$ derivatives (mass increment of 299.3 Da; data not shown).

Conservation of $\operatorname{Trp}$ residues in $\mathbf{L d t}_{\mathrm{fm}}$ homologues. The Trp residues were poorly conserved in L,Dtranspeptidases from Gram-positive and Gram-negative bacteria (Supplementary Fig. S3), as expected from the substantial high residual acylation activity of $\operatorname{Ldt}_{\mathrm{fm}}$ derivatives containing $\operatorname{Trp}$ to Phe substitutions (above, Fig. $2 \mathrm{~A}$ and $\mathrm{B}$ ). However, residue e $\left(\operatorname{Trp}^{425}\right)$ was relatively conserved in L,Dtranspeptidases from $M$. tuberculosis, being present in the Mt1, Mt2, Mt3, and Mt4 enzymes as well as in the corresponding orthologues of $M$. abscessus. These enzymes are known to display bi-phasic fluorescence kinetics upon inactivation by carbapenems ${ }^{23-24,29}$ and (unpublished results). Since Trp ${ }^{425}$ was the only conserved Trp residue in the LDTs from mycobacteria we investigated the impact of a Trp to Phe substitution at this position in Ldt $_{\mathrm{fm}}$. As shown in Supplementary Fig. S4A and B, derivatives of $\mathrm{Ldt}_{\mathrm{fm}}$ lacking all Trp residues except $\operatorname{Trp}^{425}$ or lacking only this residue displayed biphasic kinetics. These results indicated that the biphasic nature of fluorescence kinetics does not depend upon any conserved Trp residue in the L,D-transpeptidase protein family.

Fluorescence kinetics with a single ectopic Trp residue at position 383. All six Trp residues of Ldt $\mathrm{fm}_{\mathrm{m}}$ were replaced by Phe residues. As expected, the variant devoid of Trp residues was very weakly fluorescent (data not shown). No modification of this weak fluorescence was observed upon acylation 
by imipenem. This observation ruled out the formal possibility that the catalytic Cys acylated by imipenem could be a fluorophore. Replacement of Tyr at position 383 by a Trp residue in this $\mathrm{Ldt}_{\mathrm{fm}}$ derivative restored biphasic fluorescence kinetics (Supplementary Fig. S4C). This result confirmed that biphasic fluorescence kinetics are not dependent upon the presence of any of the six Trp residues of Ldt $_{\mathrm{fm}}$ in their original positions.

Investigation of the opened $\beta$-lactam ring linked to the catalytic Cys residue as a quencher. Since the variations in the fluorescence observed during the two steps of the acylation reaction could not be accounted for by modification of the environment of any specific Trp residue, our next objective was to investigate whether quenching could result from energy transfer from Trp residues to the Cys- $\beta$ lactam covalent adduct generated upon enzyme inactivation. To investigate this possibility, we first examined acylation of $\mathbf{L d t}_{\mathrm{fm}}$ by the chromogenic cephalosporin nitrocefin. Fluorescence kinetics with this compound displayed a monophasic behavior with a large decrease $(72 \%)$ in the fluorescence intensity (Fig. $3 \mathrm{~A}$ ). In the $\mathrm{Ldt}_{\mathrm{fm}}$-nitrocefin acylenzyme, the negative charge developing on the $\beta$-lactam nitrogen is stabilized by a mesomeric effect that prevents protonation of the amine ${ }^{30}$. Thus, the amine anion formed as an end product with nitrocefin (Fig. 3A) or as an intermediate with imipenem (Fig. 1) was associated with extensive fluorescence quenching. As found for nitrocefin, acylation of $\mathrm{Ldt}_{\mathrm{fm}}$ by ceftriaxone led to a monophasic decrease in the fluorescence intensity (Fig. 3B). Acylation of $\mathrm{Ldt}_{\mathrm{fm}}$ by ceftriaxone involves a concerted mechanism leading to the elimination of the side chain and formation of a conjugated imine ${ }^{7}$. The extent of fluorescence quenching observed for the acylenzyme formed with ceftriaxone (40\%; imine) or by the intermediate formed with imipenem (45\%; amine anion) were more important than that observed for the final $L_{d t}$ fm-imipenem adduct $(20 \%)$ suggesting that quenching was decreased by protonation of the $\beta$-lactam nitrogen.

Fluorescence quenching associated with inactivation of $\beta$-lactamases by $\beta$-lactamase inhibitors. In order to enrich the structural diversity of molecules investigated in the fluorescence quenching assay, we aimed to test $\beta$-lactamase inhibitors. Most $\beta$-lactams could not be tested with $\beta$-lactamases since the fluorescence assay requires relatively high enzyme concentrations (typically $10 \mu \mathrm{M}$ ) leading to rapid hydrolysis of the drugs even if the turnovers are low. For this reason, we focused on inactivation of BlaC from Mycobacterium tuberculosis by clavulanate since the corresponding acylenzyme is not prone to hydrolysis at the timescale of our experiments ${ }^{31}$. Acylation of $\mathrm{BlaC}$ by clavulanate led to a large decrease in the fluorescence intensity (37\%) (Fig. 3C). Previous studies have shown that acylation of $\mathrm{BlaC}$ by clavulanate leads to formation of imine and trans-enamine tautomers (depicted in Fig. $3 \mathrm{C}$ ) following drug decarboxylation ${ }^{32}$. Since $\mathrm{Ldt}_{\mathrm{fm}}$ and $\mathrm{BlaC}$ contain Cys and Ser as the active-site nucleophile, respectively, these results indicate that fluorescence quenching is observed with acylenzymes containing both thioester and ester bonds.

Avibactam offered the possibility to test a non- $\beta$-lactam inhibitor that inhibits $\mathrm{BlaC}$ by formation of a carbamoylenzyme ${ }^{33}$. Formation of the adduct (Supplementary Fig. S5) was not associated with any significant modification of the fluorescence intensity of the enzyme (Fig. 3D). Protonation of the amino sulfate nitrogen of avibactam in the avibactam-BlaC adduct was thus associated with limited quenching as found for the protonation of the $\beta$-lactam nitrogen in the final $\mathrm{Ldt}_{\mathrm{fm}}$-imipenem and BlaCclavulanate adducts (Fig. 1 and 3C).

Acylation of $\mathbf{L d t}_{\mathrm{fm}}$ and BlaC by faropenem. Faropenem, a $\beta$-lactam belonging to the penem class, is poorly hydrolyzed by $\mathrm{BlaC}$ and rapidly inactivates $\mathrm{L}, \mathrm{D}$-transpeptidases ${ }^{24}$. Thus, faropenem provided the opportunity to study the acylation of the two enzyme types, i.e. a $\beta$-lactamase (BlaC) and an L,D- 
transpeptidase $\left(\mathrm{Ldt}_{\mathrm{fm}}\right)$, by the same $\beta$-lactam. Acylation of BlaC by faropenem led to extensive fluorescence quenching (44\%) (Fig. 3E). The resulting acylenzyme contained an unprotonated $\beta$-lactam nitrogen. In contrast, acylation of $\mathrm{Ldt}_{\mathrm{fm}}$ by faropenem, which resulted in the rupture of the $\mathrm{C}^{5}-\mathrm{C}^{6}$ bond and the loss of a large portion of the drug including the $\beta$-lactam nitrogen ${ }^{24}$, was associated with a moderate decrease in the fluorescence intensity (17\%) (Fig. 3F).

Acylation of BlaC by the meropenem carbapenem. Although BlaC displays moderate carbapenemase activity, previous studies have shown that a BlaC-meropenem adduct is the predominant enzyme form upon incubation of the enzyme with low drug concentrations ${ }^{34-35}$. As expected, the control experiment depicted in Supplementary Fig. 66 shows that the concentration of meropenem remains saturating for at least 80 min during hydrolysis of meropenem $(40-200 \mu \mathrm{M})$ by BlaC $(10 \mu \mathrm{M})$. Fluorescence kinetics at a shorter time scale (6 min) showed a transitory decrease in fluorescence intensity (40\%) (Fig. 3G). This observation suggests that formation of an amine anion initially leads to an important fluorescence quenching. Then, fluorescence returned to the initial level suggesting that protonation of the amine anion fully suppressed quenching. Thus, formation of an amine anion following nucleophilic attack of the carbonyl carbon of carbapenems by Ldt $_{\text {fm }}$ (Fig. 1) and BlaC (Fig. 3G) similarly led to extensive fluorescence quenching despite the presence of different catalytic residues (Cys versus Ser) and different modes of activation of these nucleophiles involving His and Lys residues.

\section{DISCUSSION}

The main aim of the present study was to determine the basis for the variations in the fluorescence intensity observed upon acylation of LDTs by $\beta$-lactam antibiotics. Previous analyses of the D,Dtranspeptidase R61 (PBP family) have shown that acylation of the enzyme by penicillin $\mathrm{G}$ leads to a fluorescence quenching, which specifically depends upon one of two Trp residues of the protein ${ }^{36-37}$. This residue, $W^{233}$, is essential for the $D, D$-carboxypeptidase activity of the protein, as determined by the release of the terminal D-Ala residue from a model peptide ending in D-Ala-D-Ala ${ }^{37}$. The crystal structure of R61 in complex with a peptide mimicking the acyl donor revealed hydrophobic interactions between the side-chain of $\mathrm{W}^{233}$ and methylene groups of this substrate ${ }^{38}$. Likewise, the structure of a penicillin-R61 complex revealed that $\mathrm{W}^{233}$ is in hydrophobic interaction with the phenylacetyl side chain of the drug ${ }^{39}$ indicating that fluorescence quenching may depend upon this direct interaction. In contrast, we show here that the variations in fluorescence during acylation of LDTs by $\beta$-lactam antibiotics require at least one Trp residue but that the position of this residue is not determinant. This was established by deleting Trp residues from Ldt $_{f m}$ in various combinations (Fig. 1 and 2), by introducing a single Trp residue in an ectopic position of Ldt $_{\mathrm{fm}}$ (Fig. S4), and by showing that large variations in fluorescence intensity occur in distantly related LDTs and unrelated $\beta$-lactamases that do not share any conserved Trp residue (Fig. S3). Since fluorescence quenching was not mainly due to changes in the environment of Trp residues, we investigated the possibility that the nucleophilic attack of the $\beta$-lactam ring could itself generate a fluorescence quencher. If this is the case, the fluorescence profile should depend upon the structural features of the drug bound to the active-site residue. Accordingly, extensive quenching was not mainly determined by the size of the side chains of $\beta$-lactams or by the nature of the enzyme nucleophile (Cys versus Ser in $\mathrm{Ldt}_{\mathrm{fm}}$ and BlaC, respectively) (Fig .3). Acylation of Ser or Cys per se was not sufficient for fluorescence quenching since a modest or no decrease in fluorescence intensity was observed for the final acylation products of $\mathrm{Ldt}_{\mathrm{fm}}$ by faropenem, of BlaC by avibactam, and of both enzymes by carbapenems. By elimination, these results pointed to 
the status of the $\beta$-lactam nitrogen as the only source of variation that could account for the main variations in fluorescence intensity. $A$ large fluorescence quenching occurred if the $\beta$-lactam nitrogen was negatively charged, as transiently found during acylation of $\mathrm{Ldt}_{\mathrm{fm}}$ and $\mathrm{BlaC}$ by carbapenems. A large fluorescence quenching also occurred if the nitrogen atom was engaged in a double bond as observed for the acylenzymes formed by $\mathrm{Ldt}_{\mathrm{fm}}$ with ceftriaxone or nitrocefin and by $\mathrm{BlaC}$ with faropenem or clavulanate. In agreement with the critical role of the double bond, fluorescence quenching was not observed for acylation of BlaC by avibactam, which results in the formation of a carbamoyl-enzyme with a protonated nitrogen, or for acylation of $\mathbf{L d t}_{\mathrm{fm}}$ by faropenem, which results in the formation of an acylenzyme lacking the nitrogen atom following rupture of the $\mathrm{C}^{5}-\mathrm{C}^{6}$ bond. These results indicate that fluorescence kinetics can provide information not only on the efficacy of enzyme inactivation but also on the structure of the covalent adducts, including for example the presence of $\Delta 1$ - or $\Delta 2$-pyrroline ring in enzyme-carbapenem adducts. Our results also indicate that nitrocefin provides a very sensitive assay to titrate the active site of enzymes that do not hydrolyze this cephalosporin. This property could be exploited to identify $\beta$-lactams and non- $\beta$-lactam inhibitors that reversibly or irreversibly bind to LDTs.

\section{EXPERIMENTAL SECTION}

Enzyme production and purification. The catalytic domain of $\mathrm{Ldt}_{\mathrm{fm}}$ (residues 341 to 466 ) and soluble $\mathrm{BlaC}$ (residues 39 to 306 ) were produced in E. coli and purified by metal affinity and size exclusion chromatography as previously described ${ }^{30}$. Synthetic genes were purchased for production of $\operatorname{Ldt}_{\mathrm{fm}}$ derivatives with Trp to Phe and His to Ala substitutions (GeneCust). All experiments were performed with a fixed concentration of $\mathrm{Ldt}_{\mathrm{fm}}$ and $\mathrm{BlaC}(10 \mu \mathrm{M})$.

Spectrofluorimetry. Fluorescence kinetics were studied in $100 \mathrm{mM}$ sodium phosphate $(\mathrm{pH} 6.0)$ at $20^{\circ} \mathrm{C}$ by using a stopped-flow apparatus (RX-2000, Applied Biophysics) coupled to a spectrofluorimeter (Cary Eclipse; Varian). Excitation was performed at $224 \mathrm{~nm}$ with a $5 \mathrm{~nm}$ slit and a $2 \mathrm{~mm}$ optical path. Fluorescence emission was determined at $335 \mathrm{~nm}$ with a $5 \mathrm{~nm}$ slit and a $10 \mathrm{~mm}$ optical path. Fluorescence data were collected exactly in the same conditions for comparison of the relative fluorescence of wild-type $\mathrm{Ldt}_{\mathrm{fm}}$ and derivatives with Trp to Phe and His to Ala substitutions. These conditions included the photomultiplier voltage, which was set to $600 \mathrm{~V}$. Kinetic constants were determined using the DynaFit software (BioKin $L t d){ }^{40}$ for each Ldt $_{\mathrm{fm}}$ derivative by simultaneously fitting the fluorescence data for the various inhibitor concentrations to differential equations derived from the reaction scheme depicted in Fig. $1 \mathrm{~A}$.

Mass spectrometry. Formation of the proposed $\mathrm{Ldt}_{\mathrm{fm}}-\beta$-lactam and BlaC- $\beta$-lactam adducts in the conditions described in the text was systematically checked by mass spectrometry (data not shown). Enzymes and $\beta$-lactams were incubated for appropriate time periods at $20^{\circ} \mathrm{C}$. Five $\mu \mathrm{l}$ of acetonitrile and $1 \mu \mathrm{l}$ of $1 \%$ formic acid were extemporaneously added. Injection in the mass spectrometer (Qstar Pulsar I; Applied Biosystem) was performed at a flow rate of $0.05 \mathrm{ml} / \mathrm{min}$ (acetonitrile, $50 \%$, water, $49.5 \%$, and formic acid, $0.5 \%$; per volume). Spectra were acquired in the positive mode, as previously described ${ }^{8}$. Detailed analyses of the mass spectra of the $\beta$-lactam adducts considered in the current study have been previously published ${ }^{7-8,24,29,41}$.

\section{ANCILLARY INFORMATION}


Supporting Information. Supplementary figures S1 to S6.

Corresponding Author Information. Correspondence should be addressed to jeanemmanuel.hugonnet@crc.jussieu.fr and/or michel.arthur@crc.jussieu.fr

Author Contribution. ST, ZE contributed equally to this work. MA and JEH contributed equally to this work.

Acknowledgement. We thank L. Dubost and A. Marie for technical assistance in the collection of mass spectra. This work was supported by the French National Research Agency (grant MycWall, ANR-17CE18-0010) and the Fondation pour la Recherche Médicale (grant ECO20160736080 to ZE).

\section{REFERENCES}

1. Turner, R. D.; Vollmer, W.; Foster, S. J., Different walls for rods and balls: the diversity of peptidoglycan. Mol Microbiol 2014, 91 (5), 862-74.

2. Zapun, A.; Contreras-Martel, C.; Vernet, T., Penicillin-binding proteins and beta-lactam resistance. FEMS Microbiol Rev 2008, 32 (2), 361-85.

3. Lavollay, M.; Arthur, M.; Fourgeaud, M.; Dubost, L.; Marie, A.; Veziris, N.; Blanot, D.; Gutmann, L.; Mainardi, J. L., The peptidoglycan of stationary-phase Mycobacterium tuberculosis predominantly contains cross-links generated by L,D-transpeptidation. J Bacteriol 2008, 190 (12), 4360-6.

4. Lavollay, M.; Fourgeaud, M.; Herrmann, J. L.; Dubost, L.; Marie, A.; Gutmann, L.; Arthur, M.; Mainardi, J. L., The peptidoglycan of Mycobacterium abscessus is predominantly cross-linked by L,D-transpeptidases. J Bacteriol 2011, 193 (3), 778-82.

5. Peltier, J.; Courtin, P.; El Meouche, I.; Lemee, L.; Chapot-Chartier, M. P.; Pons, J. L., Clostridium difficile has an original peptidoglycan structure with a high level of $\mathrm{N}$-acetylglucosamine deacetylation and mainly 3-3 cross-links. J Biol Chem 2011, 286 (33), 29053-62.

6. Sutterlin, L.; Edoo, Z.; Hugonnet, J. E.; Mainardi, J. L.; Arthur, M., Peptidoglycan Cross-Linking Activity of I,d-Transpeptidases from Clostridium difficile and Inactivation of These Enzymes by beta-Lactams. Antimicrob Agents Chemother 2018, 62 (1).

7. Triboulet, S.; Dubee, V.; Lecoq, L.; Bougault, C.; Mainardi, J. L.; Rice, L. B.; Etheve-Quelquejeu, M.; Gutmann, L.; Marie, A.; Dubost, L.; Hugonnet, J. E.; Simorre, J. P.; Arthur, M., Kinetic features of L,D-transpeptidase inactivation critical for beta-lactam antibacterial activity. PLoS One 2013, 8 (7), e67831.

8. Mainardi, J. L.; Hugonnet, J. E.; Rusconi, F.; Fourgeaud, M.; Dubost, L.; Moumi, A. N.; Delfosse, V.; Mayer, C.; Gutmann, L.; Rice, L. B.; Arthur, M., Unexpected inhibition of peptidoglycan LDtranspeptidase from Enterococcus faecium by the beta-lactam imipenem. J Biol Chem 2007, 282 (42), 30414-22.

9. Mainardi, J. L.; Legrand, R.; Arthur, M.; Schoot, B.; van Heijenoort, J.; Gutmann, L., Novel mechanism of beta-lactam resistance due to bypass of DD-transpeptidation in Enterococcus faecium. J Biol Chem 2000, 275 (22), 16490-6.

10. Mainardi, J. L.; Morel, V.; Fourgeaud, M.; Cremniter, J.; Blanot, D.; Legrand, R.; Frehel, C.; Arthur, M.; Van Heijenoort, J.; Gutmann, L., Balance between two transpeptidation mechanisms determines the expression of beta-lactam resistance in Enterococcus faecium. J Biol Chem 2002, 277 (39), 35801-7.

11. Hugonnet, J. E.; Mengin-Lecreulx, D.; Monton, A.; den Blaauwen, T.; Carbonnelle, E.; Veckerle, C.; Brun, Y. V.; van Nieuwenhze, M.; Bouchier, C.; Tu, K.; Rice, L. B.; Arthur, M., Factors essential 
for L,D-transpeptidase-mediated peptidoglycan cross-linking and beta-lactam resistance in Escherichia coli. Elife 2016, 5, e19469.

12. Both, D.; Steiner, E. M.; Stadler, D.; Lindqvist, Y.; Schnell, R.; Schneider, G., Structure of LdtMt2, an L,D-transpeptidase from Mycobacterium tuberculosis. Acta Crystallogr D Biol Crystallogr 2013, 69 (Pt 3), 432-41.

13. Correale, S.; Ruggiero, A.; Capparelli, R.; Pedone, E.; Berisio, R., Structures of free and inhibited forms of the L,D-transpeptidase Ldt Mt1 $_{1}$ from Mycobacterium tuberculosis. Acta Crystallogr D Biol Crystallogr 2013, 69 (Pt 9), 1697-706.

14. Li, W. J.; Li, D. F.; Hu, Y. L.; Zhang, X. E.; Bi, L. J.; Wang, D. C., Crystal structure of L,Dtranspeptidase $\mathrm{Ldt}_{\mathrm{Mt2}}$ in complex with meropenem reveals the mechanism of carbapenem against Mycobacterium tuberculosis. Cell Res 2013, 23 (5), 728-31.

15. Biarrotte-Sorin, S.; Hugonnet, J. E.; Delfosse, V.; Mainardi, J. L.; Gutmann, L.; Arthur, M.; Mayer, C., Crystal structure of a novel beta-lactam-insensitive peptidoglycan transpeptidase. J Mol Biol 2006, 359 (3), 533-538.

16. Mainardi, J. L.; Fourgeaud, M.; Hugonnet, J. E.; Dubost, L.; Brouard, J. P.; Ouazzani, J.; Rice, L. B.; Gutmann, L.; Arthur, M., A novel peptidoglycan cross-linking enzyme for a beta-lactam-resistant transpeptidation pathway. J Biol Chem 2005, 280 (46), 38146-38152.

17. Goffin, C.; Ghuysen, J. M., Biochemistry and comparative genomics of SxxK superfamily acyltransferases offer a clue to the mycobacterial paradox: presence of penicillin-susceptible target proteins versus lack of efficiency of penicillin as therapeutic agent. Microbiol Mol Biol Rev 2002, 66 (4), 702-38, table of contents.

18. Lecoq, L.; Dubee, V.; Triboulet, S.; Bougault, C.; Hugonnet, J. E.; Arthur, M.; Simorre, J. P., Structure of Enterococcus faecium L,D-transpeptidase acylated by ertapenem provides insight into the inactivation mechanism. ACS Chem Biol 2013, 8 (6), 1140-6.

19. Magnet, S.; Arbeloa, A.; Mainardi, J. L.; Hugonnet, J. E.; Fourgeaud, M.; Dubost, L.; Marie, A.; Delfosse, V.; Mayer, C.; Rice, L. B.; Arthur, M., Specificity of L,D-transpeptidases from grampositive bacteria producing different peptidoglycan chemotypes. J Biol Chem 2007, 282 (18), 13151-9.

20. Tipper, D. J.; Strominger, J. L., Mechanism of action of penicillins: a proposal based on their structural similarity to acyl-D-alanyl-D-alanine. Proc Natl Acad Sci U S A 1965, 54 (4), 1133-41.

21. Triboulet, S.; Arthur, M.; Mainardi, J. L.; Veckerle, C.; Dubee, V.; Nguekam-Nouri, A.; Gutmann, L.; Rice, L. B.; Hugonnet, J. E., Inactivation kinetics of a new target of beta-lactam antibiotics. J Biol Chem 2011, 286 (26), 22777-84.

22. Bhattacharjee, N.; Field, M. J.; Simorre, J. P.; Arthur, M.; Bougault, C. M., Hybrid potential simulation of the acylation of Enterococcus faecium L,D-transpeptidase by carbapenems. J Phys Chem B 2016, 120 (21), 4767-81.

23. Dubee, V.; Triboulet, S.; Mainardi, J. L.; Etheve-Quelquejeu, M.; Gutmann, L.; Marie, A.; Dubost, L.; Hugonnet, J. E.; Arthur, M., Inactivation of Mycobacterium tuberculosis I,d-transpeptidase LdtMt(1) by carbapenems and cephalosporins. Antimicrob Agents Chemother 2012, 56 (8), 418995.

24. Dhar, N.; Dubée, V.; Ballell, L.; Cuinet, G.; Hugonnet, J.-L.; Signorino-Gelo, F.; Barros, D.; Arthur, M.; McKinney, J., Rapid cytolysis of Mycobacterium tuberculosis by faropenem, an orally bioavailable beta-lactam antibiotic. Antimicrob Agents Chemother 2015, 59, 1308-1319.

25. Gupta, R.; Lavollay, M.; Mainardi, J. L.; Arthur, M.; Bishai, W. R.; Lamichhane, G., The Mycobacterium tuberculosis protein $\operatorname{Ldt}(\mathrm{Mt} 2)$ is a nonclassical transpeptidase required for virulence and resistance to amoxicillin. Nat Med 2010, 16, 466-469.

26. Diacon, A. H.; van der Merwe, L.; Barnard, M.; von Groote-Bidlingmaier, F.; Lange, C.; GarciaBasteiro, A. L.; Sevene, E.; Ballell, L.; Barros-Aguirre, D., beta-lactams against tuberculosis-new trick for an old dog? N Engl J Med 2016, 375 (4), 393-4.

27. Iannazzo, L.; Soroka, D.; Triboulet, S.; Fonvielle, M.; Compain, F.; Dubee, V.; Mainardi, J. L.; Hugonnet, J. E.; Braud, E.; Arthur, M.; Etheve-Quelquejeu, M., Routes of synthesis of carbapenems for optimizing both the inactivation of L,D-transpeptidase $\operatorname{Ldt}_{\mathrm{Mt1}}$ of 
Mycobacterium tuberculosis and the stability toward hydrolysis by beta-Lactamase BlaC. $J$ Med Chem 2016, 59 (7), 3427-38.

28. Edoo, Z.; Iannazzo, L.; Compain, F.; Li de la Sierra Gallay, I.; van Tilbeurgh, H.; Fonvielle, M.; Bouchet, F.; Le Run, E.; Mainardi, J. L.; Arthur, M.; Etheve-Quelquejeu, M.; Hugonnet, J. E., Synthesis of avibactam derivatives and activity on beta-lactamases and peptidoglycan biosynthesis enzymes of mycobacteria. Chemistry 2018, 24(32), 8081-6.

29. Cordillot, M.; Dubee, V.; Triboulet, S.; Dubost, L.; Marie, A.; Hugonnet, J. E.; Arthur, M.; Mainardi, J. L., In vitro cross-linking of Mycobacterium tuberculosis peptidoglycan by L,D-transpeptidases and inactivation of these enzymes by carbapenems. Antimicrob Agents Chemother 2013, 57 (12), 5940-5.

30. Edoo, Z.; Arthur, M.; Hugonnet, J. E., Reversible inactivation of a peptidoglycan transpeptidase by a beta-lactam antibiotic mediated by beta-lactam-ring recyclization in the enzyme active site. Sci Rep 2017, 7 (1), 9136.

31. Hugonnet, J. E.; Blanchard, J. S., Irreversible inhibition of the Mycobacterium tuberculosis betalactamase by clavulanate. Biochemistry 2007, 46 (43), 11998-2004.

32. Tremblay, L. W.; Hugonnet, J. E.; Blanchard, J. S., Structure of the covalent adduct formed between Mycobacterium tuberculosis beta-lactamase and clavulanate. Biochemistry 2008, 47 (19), 5312-6.

33. Xu, H.; Hazra, S.; Blanchard, J. S., NXL104 irreversibly inhibits the beta-lactamase from Mycobacterium tuberculosis. Biochemistry 2012, 51 (22), 4551-7.

34. Hugonnet, J. E.; Tremblay, L. W.; Boshoff, H. I.; Barry, C. E., 3rd; Blanchard, J. S., Meropenemclavulanate is effective against extensively drug-resistant Mycobacterium tuberculosis. Science 2009, 323 (5918), 1215-8.

35. Tremblay, L. W.; Fan, F.; Blanchard, J. S., Biochemical and structural characterization of Mycobacterium tuberculosis beta-lactamase with the carbapenems ertapenem and doripenem. Biochemistry 2010, 49 (17), 3766-73.

36. Frere, J. M.; Ghuysen, J. M.; Iwatsubo, M., Kinetics of interaction between the exocellular DDcarboxypeptidase-transpeptidase from Streptomyces R61 and beta-lactam antibiotics. A choice of models. Eur J Biochem 1975, 57 (2), 343-51.

37. Bourguignon-Bellefroid, C.; Wilkin, J. M.; Joris, B.; Aplin, R. T.; Houssier, C.; Prendergast, F. G.; Van Beeumen, J.; Ghuysen, J. M.; Frere, J. M., Importance of the two tryptophan residues in the Streptomyces R61 exocellular DD-peptidase. Biochem J 1992, 282 ( Pt 2), 361-7.

38. McDonough, M. A.; Anderson, J. W.; Silvaggi, N. R.; Pratt, R. F.; Knox, J. R.; Kelly, J. A., Structures of two kinetic intermediates reveal species specificity of penicillin-binding proteins. J Mol Biol 2002, 322 (1), 111-22.

39. Silvaggi, N. R.; Josephine, H. R.; Kuzin, A. P.; Nagarajan, R.; Pratt, R. F.; Kelly, J. A., Crystal structures of complexes between the R61 DD-peptidase and peptidoglycan-mimetic betalactams: a non-covalent complex with a "perfect penicillin". J Mol Biol 2005, 345 (3), 521-33.

40. Kuzmic, P., DynaFit--a software package for enzymology. Methods Enzymo/ 2009, 467, 247-80.

41. Soroka, D.; Ourghanlian, C.; Compain, F.; Fichini, M.; Dubée, V.; Mainardi, J.; Hugonnet, J.; Arthur, M., Inhibition of $\beta$-lactamases of mycobacteria by avibactam and clavulanate. $J$ Antimicrob Chemother 2017, 72, 1081-1088.

42. Chow, C.; Xu, H.; Blanchard, J. S., Kinetic characterization of hydrolysis of nitrocefin, cefoxitin, and meropenem by beta-lactamase from Mycobacterium tuberculosis. Biochemistry 2013, 52 (23), 4097-104. 

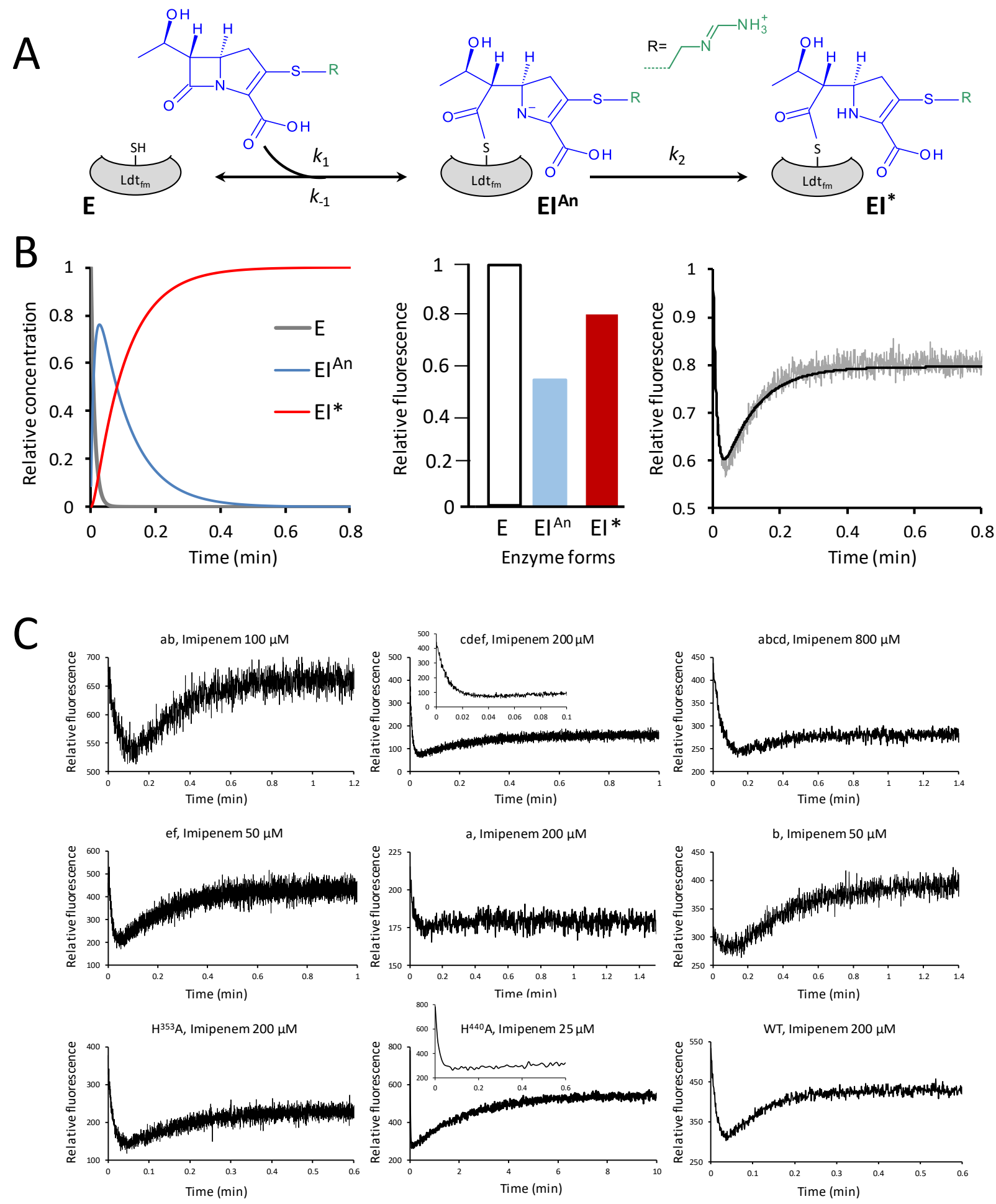

Figure 1. Impact of amino acid substitutions on fluorescence kinetics. (A) Inactivation reaction scheme. $\mathrm{E}$, free $\mathrm{Ldt}_{\mathrm{fm}} ; \mathrm{El}^{\mathrm{An}}$, amine anion; $\mathrm{El}{ }^{*}$, acylenzyme; I, imipenem. (B) Fluorescence variations during $\mathrm{Ldt}_{\mathrm{fm}}$ inactivation. The charts show the variations in the relative concentrations of the three enzyme forms (left panel) and the relative fluorescence intensity of the three enzyme forms (middle panel), which account for the observed fluorescence kinetics (right panel). (C) Fluorescence kinetics of $\mathrm{Ldt}_{\mathrm{fm}}$ inactivation by imipenem. Trp residues were replaced by Phe residues in various combinations. Designation of the remaining $\operatorname{Trp}$ residues: $a, \operatorname{Trp}^{355} ; b, \operatorname{Trp}^{385} ; c, \operatorname{Trp}^{410} ; d, \operatorname{Trp}^{415} ; \mathrm{e}$, $\operatorname{Trp}{ }^{425} ; f, \operatorname{Trp}^{434}$. Insets present the initial decreases of fluorescence over a short timescale. 
A

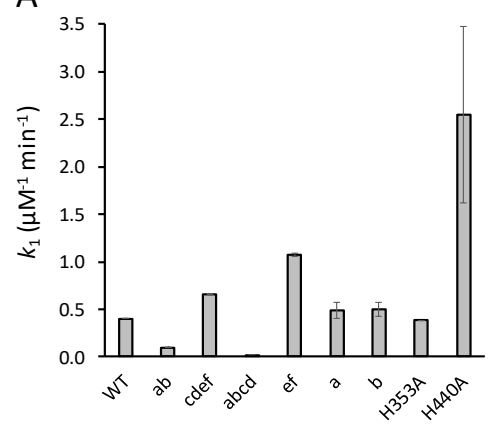

B

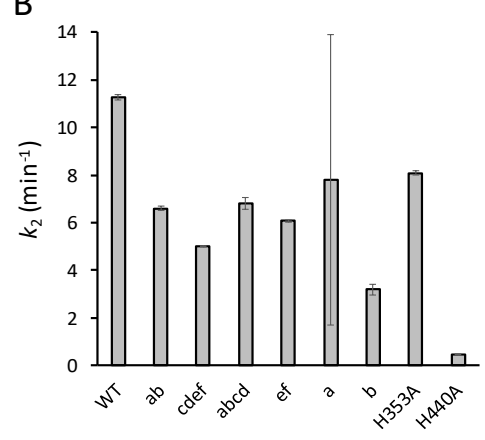

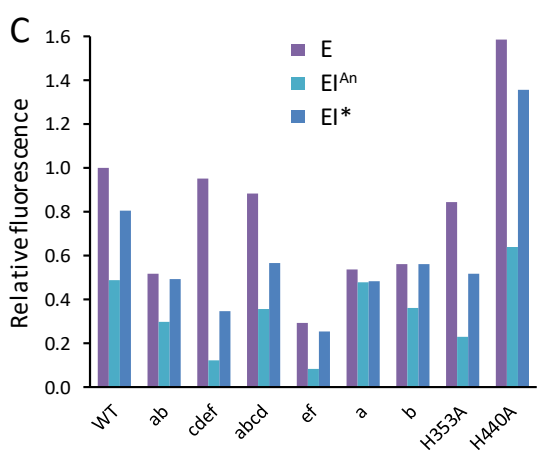

Figure 2. Inactivation efficacy and relative fluorescence intensity of $\mathbf{L d t}_{\mathrm{fm}}$ and derivatives with $\operatorname{Trp}$ to Phe and His to Ala substitutions. (A) Kinetic parameter $k_{1}$ for formation of the amine anion. (B) Kinetic parameter $k_{2}$ for the acylation step. Error bars represent standard deviations from the fitted curves. (C) Fluorescence intensity of the three enzyme forms relative to that of the free form of the wild-type enzyme. $\mathrm{E}, \mathrm{Ldt}_{\mathrm{fm}} ; \mathrm{EI}^{\mathrm{An}}$, amine anion; $\mathrm{EI}{ }^{*}$, acylenzyme. All, presence of the full complement of $\operatorname{Trp}$ residues $(a, d, c, d, e$, and $f)$. Trp residues were replaced by Phe residues in various combinations. Designation of the remaining $\operatorname{Trp}$ residues: $a, \operatorname{Trp}^{355} ; b, \operatorname{Trp}^{385} ; c, \operatorname{Trp}^{410} ; d, \operatorname{Trp}^{415} ; e, \operatorname{Trp}^{425} ; f, \operatorname{Trp}^{434}$. 

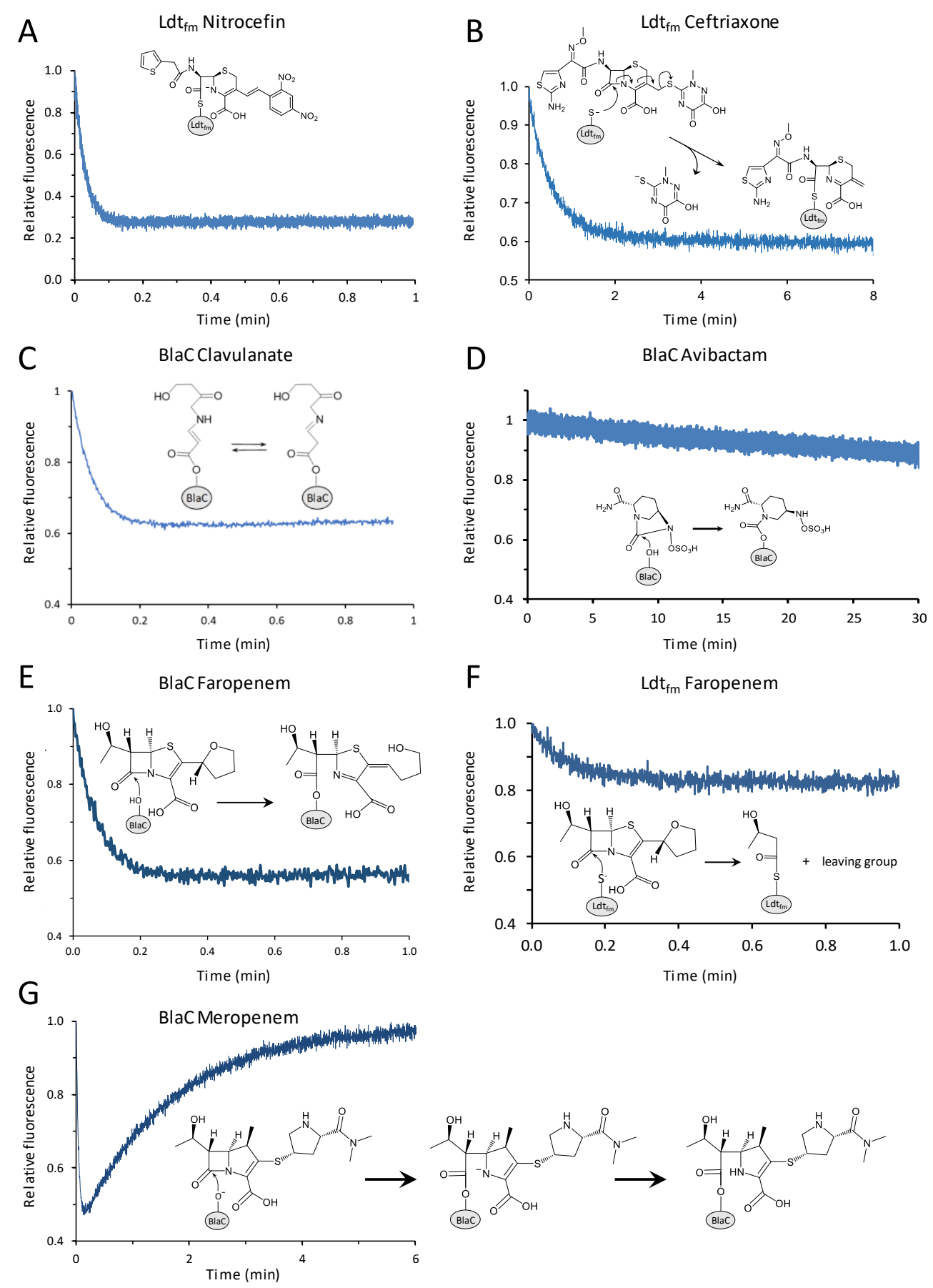

Figure 3. Variations in fluorescence intensity resulting from acylation of L,D-transpeptidase Ldt $_{\mathrm{fm}}$ and $\beta$-lactamase BlaC by various $\beta$-lactams and $\beta$-lactamase inhibitors. Stopped-flow fluorescence kinetics were obtained with final concentrations of $10 \mu \mathrm{M}$ for the enzymes and $100 \mu \mathrm{M}$ for $\beta$-lactams and $\beta$-lactamase inhibitors $(\mathbf{A})$ The negative charge developing on the amine nitrogen upon acylation of $\operatorname{Ldt}_{\mathrm{fm}}$ by nitrocefin is stabilized by a mesomeric effect involving the electron withdrawing dinitrobenzene ${ }^{30,42}$. (B) Transfer of the negative charge to a leaving group in the concerted mechanism leading to acylation of $\mathrm{Ldt}_{\mathrm{fm}}$ by ceftriaxone ${ }^{7}$. (C) Tautomeric forms of the acylenzyme resulting from acylation of BlaC by clavulanate and decarboxylation of the $\beta$-lactamase inhibitor ${ }^{32,}{ }^{41}$. (D) BlaCavibactam carbamoylenzyme ${ }^{33}$. (E and $\mathbf{F}$ ) Acylation of $\mathrm{BlaC}$ and $\mathrm{Ldt}_{\mathrm{fm}}$ by faropenem, respectively ${ }^{24}$. (G) Accumulation of an amine anion in the onset of the reaction of hydrolysis of meropenem by BlaC $\left(\mathrm{BlaC}^{\mathrm{An}}\right)$. This is followed by protonation of the $\beta$-lactam nitrogen ${ }^{35}$. 